\title{
IR spectra of the microquasar GRS 1915+105 during a low state
}

\author{
E. T. Harlaftis ${ }^{1}$, V. S. Dhillon ${ }^{2}$, and A. Castro-Tirado ${ }^{3,4}$ \\ 1 Institute of Astronomy and Astrophysics, National Observatory of Athens, PO Box 20048, Athens 118 10, \\ Greece \\ 2 Department of Physics and Astronomy, University of Sheffield, Sheffield S3 7RH, UK \\ 3 Instituto de Astrofísica de Andalucía (IAA-CSIC), PO Box 03004, 18080 Granada, Spain \\ ${ }^{4}$ Laboratorio de Astrofísica Espacial y Física Fundamental (LAEFF-INTA), PO Box 50727, 28080 Madrid, Spain
}

Received 29 December 2000 / Accepted 12 January 2001

\begin{abstract}
There is controversy regarding the nature of the suspected donor star to the microquasar GRS 1915+105, and hence whether GRS 1915+105 is a high mass X-ray binary (HMXB) or a low-mass X-ray binary (LMXB). In order to clarify this issue, we obtained an infrared $(J, H$ and $K)$ spectrum of GRS $1915+105$ in its low state which shows a steeper continuum than the prototype X-ray binary Sco X-1. We did not detect any He II emission at $2.189 \mu \mathrm{m}$ from GRS $1915+105$ in our quiescent spectrum, indicating that the line is transient and is only observed during episodes of high X-ray activity. For our instrument configuration, there is no detection of the ${ }^{12} \mathrm{CO}$ lines which are characteristic of late-type stars either in GRS $1915+105$ or Sco X-1.
\end{abstract}

Key words. infrared: stars - X-ray: stars - black hole physics - stars: binaries - stars: individual: GRS $1915+105$

\section{Introduction}

The galactic X-ray source GRS 1915+105 (Castro-Tirado et al. 1994) was the first object in our Galaxy to show superluminal jets (there are about 10 microquasars in our Galaxy now; see Mirabel \& Rodríguez 1999). Until then, apparent superluminal motion had only been observed in active galactic nuclei, which are thought to be powered by supermassive black holes. The proximity of GRS 1915+105 (12 kpc; Chaty et al. 1996) and the short-term variations it exhibits, provide an excellent testbed to investigate the detailed physics of relativistic jets. Ejection of relativistic plasma clouds in the form of synchrotron flares at infrared and radio wavelengths (Fender et al. 1997; Pooley \& Fender 1997; Eikenberry et al. 1998; Mirabel et al. 1998) results in the rapid disappearance of the inner accretion disc, following the model proposed by Belloni et al. (1997), offering the first convincing connection between accretion discs and jets. Also, GRS 1915+105 is probably the heaviest known stellar-mass black hole in the Galaxy; the $67 \mathrm{~Hz}$ QPO present in the X-rays implies a mass of $33 M_{\odot}$ for a nonrotating black hole, assuming that the QPO arises in a Keplerian orbit at the inner accretion disc (Morgan et al. 1997; Cui et al. 1998).

Send offprint requests to: E. T. Harlaftis,

e-mail: ehh@astro.noa.gr
Due to the large optical extinction towards the source, IR spectroscopy, coupled with simultaneous RXTE and radio observations, is the only possible way of gaining further insight into the nature of this exotic system. In particular, IR spectroscopy has caused some debate on the correct model of the system based on the visibility of the He II emission line at $2.189 \mu \mathrm{m}$. The $K$-band spectrum of GRS $1915+105$ shows strong He I $(2.059 \mu \mathrm{m})$ and $\mathrm{Br} \gamma$ $(2.166 \mu \mathrm{m})$ emission lines. Eikenberry et al. (1998) found flux variations of a factor of 5 in these lines on a 5 min timescale. During flares, the line fluxes varied linearly with the IR continuum flux, implying that the lines are radiatively pumped by the flares. He II $(2.189 \mu \mathrm{m})$ emission has been reported (Castro-Tirado et al. 1996; Eikenberry et al. 1998) whereas Mirabel et al. (1997) did not observe any trace of it. Based on the detection or not of the He II emission line, it was suggested that the companion could be a LMXB or a HMXB, respectively. Since the spectral type of the companion star of GRS $1915+105$ is still an open question we attempt, in this paper, to clarify the issue by revisiting the object and comparing the HK spectrum with that of the prototype X-ray binary Sco X-1.

\section{Observations}

We obtained $J(1.002-1.322 \mu \mathrm{m}), H(1.455-2.094 \mu \mathrm{m})$ and $K$-band $(1.906-2.547 \mu \mathrm{m})$ spectra of GRS $1915+105$ 
Table 1. Journal of observations ( $t_{\exp }$ is the total time exposure, $\lambda_{\text {cen }}$ is the central wavelength of the band observed and UTC refers to the time at mid-exposure)

\begin{tabular}{lcccccc}
\hline \hline Star & $t_{\exp }$ & $\lambda_{\text {cen }}=2.227 \mu \mathrm{m}$ & $t_{\exp }$ & $\lambda_{\text {cen }}=1.775 \mu \mathrm{m}$ & $t_{\exp }$ & $\lambda_{\text {cen }}=1.162 \mu \mathrm{m}$ \\
\hline Sco X-1 & $\min$ & UTC & $\min$ & UTC & $\min$ & UTC \\
GRS 1915+105 & 16.0 & $06: 20: 00$ & 24.0 & $10: 09: 39$ & 16.0 & $05: 42: 25^{\dagger}$ \\
DM +49 3718 & 64.0 & $07: 56: 59$ & 80.0 & $11: 34: 10$ & 64.0 & $13: 48: 27$ \\
BS 7315 & 10.7 & $09: 01: 51$ & 5.3 & $09: 32: 08$ & 8.0 & $14: 47: 13$ \\
\hline
\end{tabular}

† Observed on 1999 August 14. All other spectra were obtained on 1999 July 8.

using the Cooled Grating Spectrometer (CGS4) on the $3.8 \mathrm{~m}$ United Kingdom Infrared Telescope (UKIRT) on Mauna Kea during the night of 1999 July 8 (journal of observations in Table 1). The 40 lines $\mathrm{mm}^{-1}$ grating was used with the $150 \mathrm{~mm}$ camera and the $256 \times 256$ pixel $\mathrm{InSb}$ array, giving resolutions of 324,426 and $340 \mathrm{~km} \mathrm{~s}^{-1}$ in the $J, H$ and $K$-bands, respectively. We also obtained $J H K$ spectra of Sco X-1 and the Be star DM +49 3718 in order to compare them with the spectral slope and emission line properties of GRS $1915+105$. The target spectra were bracketed by observations of nearby F-type stars, which were used during the data reduction to remove the telluric atmospheric features. We utilized the non-destructive readout mode to minimize the readout noise. The spectra were obtained with the one pixel slit (1.23 arcsec) and were sampled over two pixels by mechanically shifting the array in 0.5 pixel steps in the dispersion direction. We exposed for a maximum of $2 \mathrm{~min}$ on the object and then shifted the spectrum onto a different spatial position on the array (by nodding the target along the slit) in order to provide accurate sky subtraction. The nodding was repeated until a good signal to noise ratio was achieved for the target spectrum. The CGS4 data reduction system performed the initial data reduction steps (application of the bad pixel mask, dark subtraction, bias subtraction, flat-field division, sky subtraction; see also Daly \& Beard 1994). The residual sky background was then removed and the object spectra were optimally extracted using FIGARO. Ripples with a periodicity of two pixels, resulting from the mechanical shifting of the array, were visible in the one-dimensional spectra and were subsequently removed (using IRFLAT in FIGARO as well as private software, for comparison). Wavelength calibration was performed using an Argon arc in the $K$-band, a Xenon arc in the $H$-band and a Krypton arc in the $J$-band, giving a rootmean-square error of $\sim 1 \AA$ with a second order polynomial fit. Flux calibration was performed using the F-type standards. The target spectra were divided by the closest observed F star spectra (with the stellar features interpolated across), and then multiplied by the flux of the standard at each wavelength, determined from black body functions with the same effective temperature and flux as the standard. The $H$-band and $K$-band spectra overlap in the region between $1.906-2.094 \mu \mathrm{m}$ and we combined them, after matching the flux scale, by averaging them using pixel weights which optimized the signal-to-noise ratio.

\section{A High mass or a Low mass $\mathrm{X}$-ray binary?}

In Fig. 1 we present the $J H K$ spectra of the $\mathrm{Be}$ star DM +49 3718, Sco X-1 and the HK spectrum of GRS $1915+105$ (the $J$-band spectrum was not useful due to the large extinction), and the F0IV-type star BS 7315 for comparison. For clarity, we show just the $K$-band spectra in Fig. 2. In Table 2, we list the wavelengths, line fluxes and equivalent widths of the most prominent lines identified in Fig. 1. Line fluxes have not been corrected for slit losses, hence only ratios of line fluxes can reliably be used. The line widths $(F W H M)$ of the emission lines in DM +49 3718 and GRS $1915+105$ are $610 \mathrm{~km} \mathrm{~s}^{-1}$ and $730 \mathrm{kms}^{-1}$, respectively. The line width of $\mathrm{Br} \gamma$ in GRS $1915+105$ is consistent with other measured widths during a low state - since the GRS 1915+105 activity started in $1992-\left(760,730 \mathrm{~km} \mathrm{~s}^{-1}\right.$ measured by Martí et al. 2000; and Mirabel et al. 1997, respectively). The line width increases during the IR flaring activity (1010 and $1260 \mathrm{~km} \mathrm{~s}^{-1}$ measured by Martí et al. 2000; Mirabel et al. 1997, respectively).

\section{The Be star DM +49 3718 spectrum}

The $K$-band spectrum of the Be star DM +49 3718 was classified as a peculiar $K$-band star, showing $\operatorname{Br} \gamma$ in emission and perhaps some trace of He II at 2.189 $\mu \mathrm{m}$ (Hanson et al. 1996). It has also been suggested that DM +49 3718 is the optical counterpart of the High Mass X-ray Binary

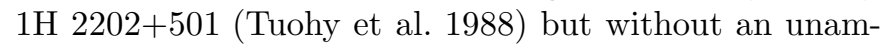
biguous confirmation so far. The equivalent width $(E W)$ of the $\mathrm{Br} \gamma$ line in our data (see Table 2) is consistent with the value of $27 \AA$ measured by Hanson et al. (1996). In our $K$ and $H$-band spectra, the Pfund and Brackett lines can individually be distinguished up to Pf28 and Br20, and the Pfund and Brackett jumps are weakly in emission at 2.279 and $1.458 \mu \mathrm{m}$, respectively. There are also emission lines at $1.069,1.087$ and $1.113 \mu \mathrm{m}$ which we identify as Si I. We identify the other emission lines at $1.129 \mu \mathrm{m}$, 1.175 and $1.317 \mu \mathrm{m}$ as O I, C I and Ca I lines (Melendez \& Barbuy 1999). The power law slope of the $J H K$ spectrum 


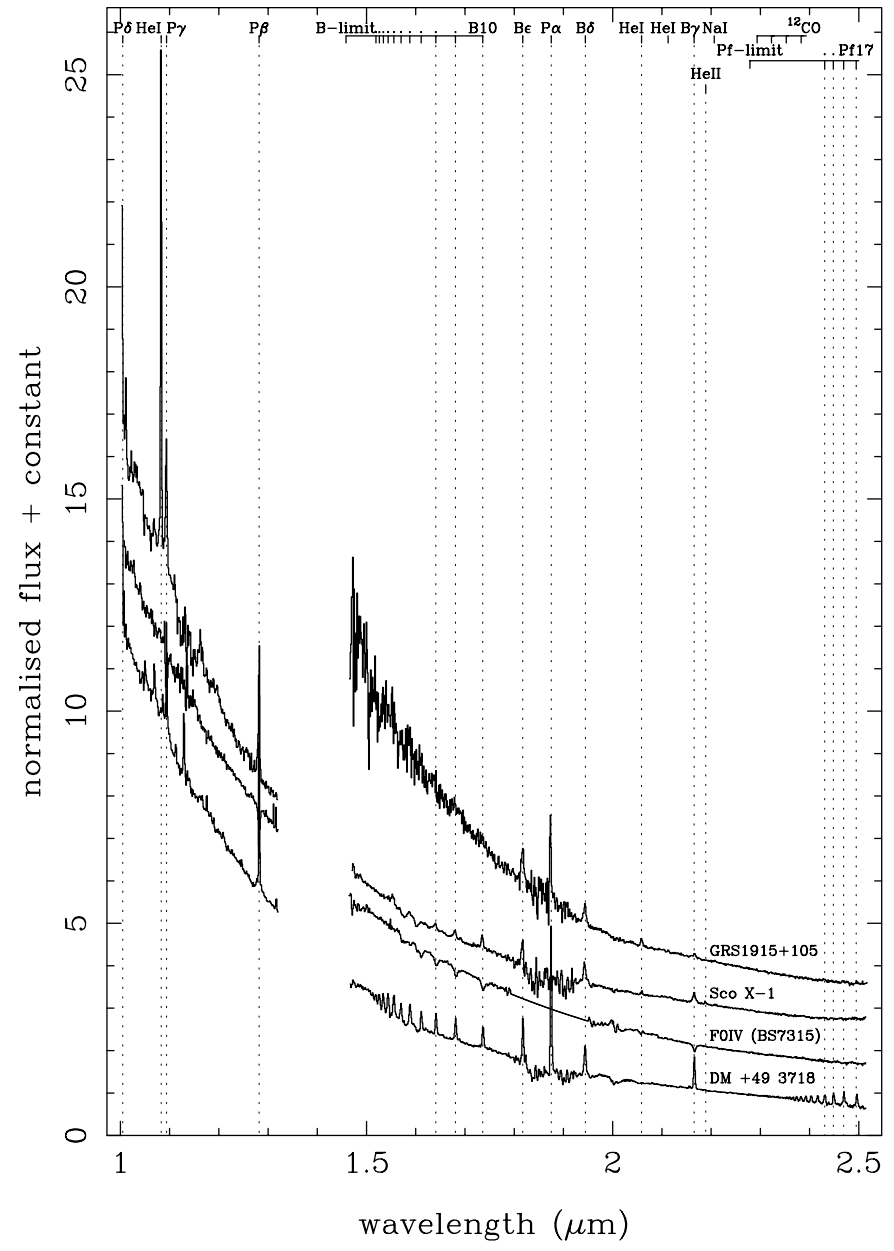

Fig. 1. The dereddened $H K$ spectrum of the microquasar GRS $1915+105$ and the $J H K$ spectra of Sco X-1 and the Be star DM +49 3718. Also plotted is the $J H K$ spectrum of the F0IV star BS 7315. The spectra have been normalised by dividing by the flux at $2.250 \mu \mathrm{m}$ and then offset on the $y$-axis by adding a multiple of 1 to each spectrum

is not significantly different from that of Sco $\mathrm{X}-1$, with a power law $\left(F_{\lambda} \propto \lambda^{\alpha}\right)$ index of $\alpha=-3.21 \pm 0.01$. The ratios of line intensities of the Pfund series are consistent with recombination case B (Hummer \& Storey 1987) within the (large) uncertainties, indicating that the line source is located in optically thin gas surrounding the high mass star.

\section{The GRS $1915+105$ spectrum}

GRS $1905+105$ is not a Wolf-Rayet star, like Cyg X-3 (van Kerkwijk et al. 1992), since we detect Br $\gamma$ in emission (see also discussion in Castro-Tirado et al. 1996) but with no absorption features from the companion star (neutral lines or molecular bands), as seen in the galactic bulge sources GX 1+4 and GX 13+1 (Bandyopadhyay et al. 1997). There is no trace of He II emission line at a time when the radio spectrum was flat and thus the disc was in a low state. The last flaring activity was observed on 1999 July 2. The radio and X-ray continua were

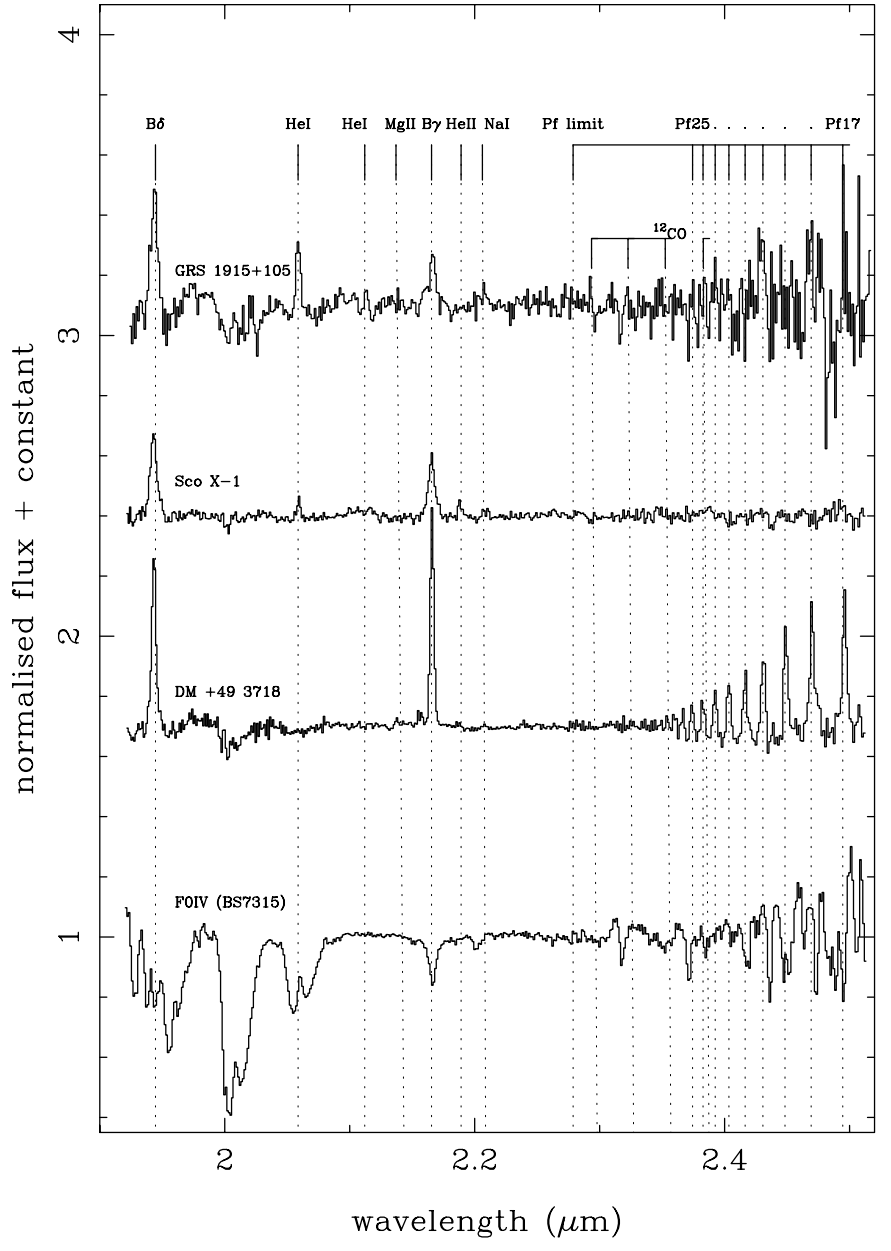

Fig. 2. The $K$-band spectra of the microquasar GRS 1915+105, Sco X-1, and the Be star DM +49 3718. Also plotted is the $K$-band spectrum of the F0IV star BS 7315 which indicates the location of telluric absorption features. The spectra have been normalized by dividing by a spline fit to their continua and then offset on the $y$-axis by adding a multiple of 0.7 to each spectrum

in their lowest states (Martí et al. 2000) which indicate that the accretion disc was quiet in terms of its behaviour since 1992. It is unclear if the He II emission is produced along the jet, in the accretion disc or on the massive star. There are indeed examples of HMXBs, such as Cyg X-1, where He II emission is produced in the stellar wind of the O9.7 Iab primary star (Gies \& Bolton 1986). However, the $\mathrm{X}$-ray burst activity is related to the jet/disc system centred on the compact object (Belloni et al. 1997), rather than on a possible massive companion star, and thereafter is the most likely cause of the increase in transient He II emission. This hypothesis is supported by the absence of He II emission during this low state, which is the time that the inner disc is being replenished by gas, suggesting that the X-ray luminosity is not sufficient to pump up the He II line through photoionization of the accretion disc.

The spectrum of GRS $1915+105$ is different from that of the Be star DM +49 3718 since there is no helium emission in the latter and the hydrogen lines have narrower 
Table 2. Wavelengths, equivalent widths $(\AA)$, and line fluxes (in units of $10^{-14}, 10^{-15}$ and $10^{-13} \mathrm{ergs} \mathrm{cm}^{-1} \mathrm{~s}^{-1}$ from left to right) of the most prominent lines in the infrared spectra of Sco X-1, GRS 1915+105, and DM +49 3718. The last two columns give the observed line intensity ratios and the theoretical case $\mathrm{B}$ (for $N_{\mathrm{e}}=10^{4} \mathrm{~cm}^{-3}, T_{\mathrm{e}}=10^{4} \mathrm{~K}$ ) line intensity ratios with respect to a line in the series $(\mathrm{Pf} 18, \operatorname{Br} 10$ and $\mathrm{P} \beta$ ). Note that the spectra have not been corrected for slit losses and hence the lines fluxes must be used with caution. The line ratios, however, are secure

\begin{tabular}{|c|c|c|c|c|c|c|c|c|c|}
\hline \multirow[b]{2}{*}{ Line } & \multirow[b]{2}{*}{$\mu \mathrm{m}$} & \multicolumn{2}{|c|}{ Sco $X-1$} & \multicolumn{2}{|c|}{ GRS $1915+105$} & \multicolumn{2}{|c|}{$D M+493718$} & \multirow[b]{2}{*}{ Int.ratio } & \multirow[b]{2}{*}{ CaseB } \\
\hline & & line flux & $E W$ & line flux & $E W$ & line flux & $E W$ & & \\
\hline $\mathrm{P} \delta$ & 1.0049 & $11.2 \pm 1.4$ & $-9 \pm 2$ & & & $13 \pm 2$ & $-7 \pm 1$ & $0.30 \pm 0.04$ & 0.34 \\
\hline He I & 1.0830 & $33.3 \pm 0.7$ & $-26 \pm 2$ & & & - & - & & \\
\hline $\mathrm{P} \gamma$ & 1.0938 & $11.3 \pm 0.6$ & $-11 \pm 1$ & & & $11 \pm 5$ & $-6 \pm 1$ & $0.26 \pm 0.06$ & 0.55 \\
\hline $\mathrm{P} \beta$ & 1.2818 & $10.1 \pm 0.3$ & $-13 \pm 1$ & & & $22 \pm 3$ & $-23 \pm 2$ & 1.00 & 1.00 \\
\hline B20 & 1.5196 & & & & & $1.0 \pm 0.3$ & $-3 \pm 2$ & $0.19 \pm 0.09$ & 0.13 \\
\hline B19 & 1.5265 & & & & & $1.5 \pm 0.3$ & $-4 \pm 2$ & $0.31 \pm 0.09$ & 0.15 \\
\hline B18 & 1.5346 & & & & & $2.2 \pm 0.3$ & $-5 \pm 3$ & $0.46 \pm 0.09$ & 0.17 \\
\hline B17 & 1.5443 & & & & & $2.4 \pm 0.3$ & $-6 \pm 3$ & $0.62 \pm 0.11$ & 0.20 \\
\hline B16 & 1.5561 & & & & & $3.4 \pm 0.3$ & $-8 \pm 3$ & $0.69 \pm 0.12$ & 0.24 \\
\hline B15 & 1.5705 & & & & & $2.4 \pm 0.3$ & $-6 \pm 3$ & $0.54 \pm 0.09$ & 0.29 \\
\hline B14 & 1.5885 & & & & & $3.4 \pm 0.3$ & $-9 \pm 3$ & $0.73 \pm 0.11$ & 0.36 \\
\hline B13 & 1.6114 & & & & & $1.9 \pm 0.2$ & $-5 \pm 2$ & $0.65 \pm 0.10$ & 0.45 \\
\hline B12 & 1.6412 & $1.0 \pm 0.2$ & $-3 \pm 1$ & & & $2.6 \pm 0.3$ & $-7 \pm 2$ & $0.77 \pm 0.12$ & 0.57 \\
\hline B11 & 1.6811 & $1.4 \pm 0.2$ & $-4 \pm 1$ & & & $3.1 \pm 0.3$ & $-9 \pm 2$ & $0.85 \pm 0.12$ & 0.75 \\
\hline B10 & 1.7367 & $2.1 \pm 0.2$ & $-7 \pm 1$ & & & $4.1 \pm 0.3$ & $-12 \pm 2$ & 1.00 & 1.00 \\
\hline $\mathrm{B} \epsilon$ & 1.8181 & $3.0 \pm 0.4$ & $-11 \pm 2$ & $3.1 \pm 0.4$ & $-18 \pm 2$ & $3.3 \pm 0.3$ & $-24 \pm 2$ & $2.34 \pm 0.25$ & 1.39 \\
\hline $\mathrm{P} \alpha$ & 1.8751 & & & & & $18 \pm 2$ & $-72 \pm 10$ & $1.65 \pm 0.44$ & 2.05 \\
\hline $\mathrm{B} \delta$ & 1.9451 & $4.1 \pm 0.2$ & $-15 \pm 2$ & $3.3 \pm 0.2$ & $-15 \pm 2$ & $5.6 \pm 0.5$ & $-23 \pm 2$ & $2.18 \pm 0.25$ & 1.99 \\
\hline He I & 2.0587 & $0.5 \pm 0.1$ & $-2.4 \pm 1$ & $0.9 \pm 0.2$ & $-9 \pm 1$ & - & - & & \\
\hline $\mathrm{B} \gamma$ & 2.1661 & $2.2 \pm 0.1$ & $-15 \pm 1$ & $1.1 \pm 0.1$ & $-14 \pm 1$ & $4.6 \pm 0.2$ & $-28 \pm 1$ & $2.73 \pm 0.15$ & 3.03 \\
\hline He II & 2.1891 & $0.4 \pm 0.1$ & $-1.6 \pm 1$ & 0 & 0 & $0.03 \pm 0.08$ & $-0.3 \pm 1.0$ & & \\
\hline Pf25 & 2.3744 & & & & & $0.5 \pm 0.2$ & $-2 \pm 2$ & $0.24 \pm 0.16$ & 0.39 \\
\hline Pf24 & 2.3828 & & & & & $0.6 \pm 0.2$ & $-3 \pm 2$ & $0.28 \pm 0.17$ & 0.44 \\
\hline Pf23 & 2.3925 & & & & & $0.7 \pm 0.2$ & $-4 \pm 2$ & $0.38 \pm 0.16$ & 0.49 \\
\hline Pf22 & 2.4035 & & & & & $0.8 \pm 0.2$ & $-6 \pm 2$ & $0.49 \pm 0.20$ & 0.56 \\
\hline Pf21 & 2.4164 & & & & & $1.0 \pm 0.2$ & $-9 \pm 2$ & $0.54 \pm 0.25$ & 0.64 \\
\hline Pf20 & 2.4314 & & & & & $1.4 \pm 0.3$ & $-11 \pm 2$ & $0.74 \pm 0.26$ & 0.73 \\
\hline Pf19 & 2.4490 & & & & & $1.8 \pm 0.3$ & $-15 \pm 2$ & $1.00 \pm 0.36$ & 0.85 \\
\hline Pf18 & 2.4700 & & & & & $2.7 \pm 0.3$ & $-19 \pm 3$ & 1.00 & 1.00 \\
\hline Pf17 & 2.4953 & & & & & $2.4 \pm 0.4$ & $-19 \pm 3$ & $1.14 \pm 0.25$ & 1.19 \\
\hline
\end{tabular}

velocity widths and larger equivalent widths. Although hydrogen and helium emission is also observed in Be HMXBs, we do not detect their characteristic neutral or molecular emission lines of $\mathrm{Na} \mathrm{I}(2.206$ and $2.209 \mu \mathrm{m})$, $\mathrm{Mg}$ I $(2.281 \mu \mathrm{m})$, and ${ }^{12} \mathrm{CO}$ bandheads (2.294, 2.323, 2.353, 2.383 $\mu \mathrm{m}$ (Everall et al. 1993). The latter neutral and molecular lines are observed in absorption in LMXBs and can determine the spectral type of the companion star as in GX $13+1$ and G1+4 (Bandyopadhyay et al. 1999). Our $K$ spectrum of GRS $1915+105$ does not show any evidence of the above trace lines either in absorption or in emission, hindered by inadequate signal-to-noise ratio. A $V L T$ spectrum on 4 July shows the trace lines of $\mathrm{Na}$ I and
Mg I in emission (Martí et al. 2000). Unfortunately, the wavelength coverage was short of the ${ }^{12} \mathrm{CO}$ bandheads. A higher-resolution VLT spectrum on 20 and 24 July shows unequivocably the ${ }^{12} \mathrm{CO}$ bandheads in absorption indicating that the companion star is a G-M spectral-type star (Greiner et al. 2001). Apparently, the system has significant transient continuum and line emission which either dilutes or fills in the late-type's photospheric lines out of detection making the detection of the late-type star so elusive until now.

We dereddened the GRS $1915+105$ using $E(B-V)=$ 9.6 (Boër et al. 1996), $A_{V}=26.5$ (Chaty et al. 1996) and the extinction law from Howarth (1983). There was 
insufficient signal in the $J$-band, given the large extinction, though after smoothing it was possible to combine the $J$-band spectrum with the $H$ and $K$-band spectra to derive a power law index of $-5.15 \pm 0.02$. The steeper slope in GRS 1915+105 than in the Be star DM +49 3718 indicates that there is a significant $J$-band emission component due to the accretion disc during this low state. For comparison, the dominant IR component is due to synchrotron emission during flares (Mirabel et al. 1998).

\section{Conclusions}

Our comparison of the infrared spectra of the microquasar GRS $1915+105$ and the prototype X-ray binary Sco X-1 give the following results. The spectra of Sco $\mathrm{X}-1$ and GRS $1915+105$ are very similar. The main difference between the Sco X-1 and GRS 1915+105 spectra is the He II line at $2.189 \mu \mathrm{m}$. In Sco X-1, the He II line is persistent and is most likely related to irradiation of the accretion disc around the compact object. In GRS $1915+105$, the He II emission line is transient and is observed only during the X-ray bursting activity (Martí et al. 2000). The other difference between the Sco X-1 and GRS $1915+105 K$-band spectra is the weaker He I line at $2.058 \mu \mathrm{m}$, which indicates a higher luminosity class for the donor star to GRS 1915+105. Finally, we find a steeper $J H K$ continuum slope in GRS $1915+105$ than in Sco $\mathrm{X}-1$. The detection of the ${ }^{12} \mathrm{CO}$ bandheads in absorption (Greiner et al. 2001) is consistent with the constraints provided by Eikenberry \& Bandyopadhyay (2001) for a Roche-lobe overflow from a late-type companion star to GRS $1915+105$. The non-detection of these bands with our UKIRT spectrum may be related, not only to the lower signal-to-noise and lower spectral resolution, but also to the transient nature of the emission lines and the state the system was during our observations. Neutral spectral features have recently been detected by Chandra which may be related to cold material surrounding GRS $1915+105$ (Lee et al. 2001), and therefore the origin of the ${ }^{12} \mathrm{CO}$ bandheads will be unequivocably attributed to a low mass star only after phase-resolved spectra show the expected Doppler shifts of the bandheads due to the orbital motion.

Acknowledgements. The data reduction and analysis was partly carried out at the Sheffield STARLINK node. Use of software developed by T. R. Marsh is gratefully acknowledged. The SERVICE programme of UKIRT was used to obtain the $J$-band spectrum of Sco X-1 and we thank Tom Kerr for observing it.

\section{References}

Bandyopadhyay, R., Shahbaz, T., Charles, P. A., van Kerkwijk, M. H., \& Naylor, T. 1997, MNRAS, 285, 718
Bandyopadhyay, R., Shahbaz, T., Charles, P. A., \& Naylor, T. 1999, MNRAS, 306, 417

Belloni, T., Méndez, M., King, A. R., van der Klis, M., \& van Paradijs J. 1997, ApJ, 479, L145

Boër, M., Greiner, J., \& Motch, C. 1996, A\&A, 305, 835

Bradshaw, C. F., Fomalont, E. B., \& Geldzahler, B. J. 1999, ApJ, 512, L121

Castro-Tirado, A. J., Geballe, T. R., \& Lund, N. 1996, ApJ, 461, L99

Castro-Tirado, A. J., Brandt, S., Lund, N., et al. 1994, ApJS, 92, 469

Castro-Tirado, A. J., Davies, J., Brandt, S., \& Lund, N. 1993, IAU Circ., 5830

Chaty, S., Mirabel, I. F., Duc, P.-A., Wink, J. E., \& Rodriguez, L. F. 1996, A\&A, 310, 825

Crampton, D., Cowley, A. P., Hutchings, J. B., \& Kaat, C. 1976, ApJ, 207, 907

Cui, W., Zhang, S. N., \& Chen, W. 1998, ApJ, 492, L53

Daly, P. N., \& Beard, S. M. 1994, CGS4/IRCAM4 Users' Guide, SUN/27, Starlink Project

Eikenberry, S. S., Matthews, K., Morgan, E. H., Remillard, R. A., \& Nelson, R. W. 1998, ApJ, 494, L61

Eikenberry, S. S., \& Bandyopadhyay, R. 2000, ApJ, in press

Everall, C., Coe, M. J., Norton, A. J., Roche, P., \& Unger, S. J. 1993, MNRAS, 262, 57

Hanson, M. M., Conti, P. S., Rieke, M. J. 1996, ApJS, 107, 281

Howarth, I. D. 1983, MNRAS, 203, 301

Hummer, D. G., \& Storey, P. J. 1987, MNRAS, 224, 801

Fender, R. P., Pooley, G. G., Brocksopp, C., \& Newell, S. J. 1997, MNRAS, 290, L65

Gies, D. R., \& Bolton, C. T. 1986, ApJ, 304, 371

Greiner, J., McCaughrean, M. J., Cuby, J. G., Castro-Tirado, A. J., \& Mennickent, R. 2001, Proceedings of the Third Microquasar Workshop, Granada, ed. A. J. Castro-Tirado, J. Greiner, \& J. M. Paredes, A\&SS, in press

van Kerkwijk, M. H., Charles, P. A., Geballe, T. R., et al. 1992, Nature, 355, 703

Lasala, J., \& Thorstensen, J. R. 1985, AJ, 90, 2077

Lee, J. C., Reynolds, C. S., Fabian, A. C., \& Blackman, E. G. 2001 [astro-phys/0012111]

Martí, J., Mirabel, I. F., Chaty, S., \& Rodríguez, L. F. 2000, A\&A, 356, 943

Melendez, J., \& Barbuy, B. 1999, ApJS, 124, 527

Mirabel, I. F., \& Rodríguez, L. F. 1999, ARA\&A, 37, 409

Mirabel, I. F., Dhawan, V., Chaty, et al. 1998, A\&A, 330, L9

Mirabel, I. F., Bandyopadhyay, R., Charles, P. A., Shahbaz, T., \& Rodriguez, L. F. 1997, ApJ, 477, L45

Mirabel, I. F., \& Rodríguez, L. F. 1994, Nature, 371, 46

Mirabel, I. F., Duc, P. A., Teyssier, et al. 1993, IAU Circ., 5830

Morgan, R., Remillard, R., \& Greiner, J. 1997, ApJ, 482, 993

Morris, P. W., Eenens, P. R. J., Hanson, M. M., Conti, P. S., \& Blum, R. D. 1996, ApJ, 470, 597

Pooley, G., \& Fender, R. 1997, MNRAS, 292, 927

Vrtilek, S. D., McClintock, J. E., Seward, F. D., Kahn, S. M., \& Wargelin, B. J. 1991, ApJS, 76, 1127

Tuohy, I. R., et al. 1988, in Physics of Neutron Stars and Black Holes, ed. Y. Tanaka (Universal Academic Press), 93

Wackerling, L. R. 1970, Memoirs RAS, 73, 153

Wood, K. S., et al. 1984, ApJS, 56, 507 